\title{
Relationship of Lifestyle, Quality of Sleep with Type 2 Diabetes and Cardiovascular Risk - A Trinidadian Study
}

Nayak BS*, Sakhamuri SM, Moore I, Jordan D, Cuffie E, Forde $\mathrm{K}$, Jones $\mathrm{N}$ and Maharaj S

Department of Preclinical Sciences, The University of the West Indies at St. Augustine, Trinidad and Tobago, West Indies

\begin{abstract}
Aim

To assess the lifestyle, quality of sleep and the prevalence of cardiovascular risk in persons living with Type 2 diabetes mellitus.

\section{Methods}

Personal interviews were conducted with 150 subjects with Type 2 diabetes mellitus at various hospitals belonging to four regional health authorities in Trinidad. Participants' lifestyle, quality of sleep and cardiovascular risk had been assessed by using the modified AHA DLR adherence, Pittsburgh quality of sleep and Framingham 10 year risk for cardiovascular disease, respectively. Blood pressure, weight, $\mathrm{HbA} 1 \mathrm{c}$ values and fasting lipid profile were collected from the patient records.

\section{Results}

Approximately $50 \%$ of male participants exhibited an intermediate risk (10-20\%) for CVD within ten years. Almost $75 \%$ of women with Diabetes had a lower chance of developing cardiovascular disease. When Framingham values were compared to the lifestyle, a significant relationship was observed in females. There was an inverse relationship between the sleep scores and systolic blood pressure.

\section{Conclusion}

Poor quality of sleep is associated with uncontrolled hypertension among the diabetic patients.
\end{abstract}

Keywords: Cardiovascular diseases; Diabetes; Lifestyle; Sleep

\section{Introduction}

Trinidad and Tobago has a population of approximately 1.3

*Corresponding author: Shivananda Nayak, Department of Preclinical Sciences, The University of the West Indies at St. Augustine, Trinidad and Tobago, West Indies, Tel: +1 8686622873; E-mail: shivananda.nayak@sta.uwi.edu

Citation: Nayak BS, Sakhamuri SM, Moore I, Jordan D, Cuffie E, et al. (2016) Relationship of Lifestyle, Quality of Sleep with Type 2 Diabetes and Cardiovascular Risk - A Trinidadian Study. J Diabetes Metab Disord 3: 013.

Received: February 15, 2016; Accepted: April 19, 2016; Published: May 03, 2016

Copyright: (C) 2016 Nayak BS, et al., This is an open-access article distributed under the terms of the Creative Commons Attribution License, which permits unrestricted use, distribution, and reproduction in any medium, provided the original author and source are credited. million people [1]. Of those, $11 \%$ has diabetes, with higher prevalence in individuals of East Indian descent [1]. Whereas persons of African descent account for $33 \%$ of patients attending public health facilities and are affected with both diabetes and hypertension [2]. The country is currently ranked $5^{\text {th }}$ in the world with diabetes [1]. Also, one in every eight persons is overweight, and $80 \%$ of them have Type 2 Diabetes (T2DM) [3].

Urbanization in the past three decades has resulted in an increase in sedentary lifestyle and change in dietary habits. Despite the fact the poor lifestyle is a major influential factor of insulin resistance, there are no objective scores widely available to assess its role in the diabetes management. In addition, high carbohydrate intake will result in consistently high blood a glucose level which is the chief contributing factor towards developing insulin resistance [4]. Regular physical activity improves the body's response to insulin as this allows more glucose to be broken down by metabolism due to the increase glucose demand of the body during exercise [1]. Ultimately, a lack of physical activity will reduce the utilization of glucose, contributing to high blood glucose levels [5].

Quantity and quality of sleep can increase the diabetes risk, impact the disease control and can also lead to cardiovascular morbidity [6]. Similarly, factors such as high blood pressure, dyslipidemia, and obesity have also been claimed as the contributing factors to their increased risk of cardiovascular morbidity in these patients [7].

\section{Methods}

After obtaining the approval from the ethics committee, T2DM subjects of 18 years and above were recruited from four sites (San Fernando General Hospital, Area Hospital Point Fortin, Point Fortin Health Centre, Sangre Grande General Hospital and Eric Williams Medical Sciences Complex) across Trinidad. Subjects with debilitating illnesses which limit their ability to exercise were excluded. Data was gathered from subjects through interview style questionnaire administration. Three well-validated questionnaires were used in this study; the Modified AHA released Diet and Lifestyle Recommendations (AHA-DLR) adherence index, Pittsburgh sleep quality score and Framingham cardiovascular risk score 2008 version.

The modified AHA-DLR adherence index is a useful instrument to assess the relationship between lifestyle behaviours and health outcomes [8] including the prevalence of metabolic syndrome [6]. It contained twelve components each dealing with the diet and lifestyle. We used a nutritional facts sheet for foods consumed locally to calculate the different elements of the patient's diet such as fat and sodium intake. The overall maximum possible score for this questionnaire is 120; with higher scores portray the healthy eating and lifestyle. The Pittsburgh Sleep Quality Index (PSQI) is a useful tool to assess the quality of sleep over a one month time interval. It generates seven component scores: subjective sleep quality, sleep latency, sleep duration, habitual sleep efficiency, sleep disturbances, use of sleeping medication, and daytime dysfunction. Out of the maximum score 21, a global PSQI of 5 or more, indicates poor sleeping quality. Many international studies using the PSQI in a variety of adult populations have supported its high validity and reliability [9-11]. 
The Framingham cardiovascular risk score contains ten components with five delegated to men and five to women to assess a ten-year risk. Individuals were given a score based on factors such as age, total cholesterol levels, HDL levels, blood pressures and treatment. This score has been validated in both European and African Americans [12].

All the participants' demographic characteristics including age, sex, ethnicity, location of residence, time since T2DM diagnosis, current symptoms and treatment, and history of CVD events were obtained. Blood pressure, lipid profile and $\mathrm{HbAlc}$ values were acquired from the patients' records. The collected data was analyzed using the SPSS software package 16 for descriptive and bivariate statistics, prediction of the measurable outcome and identifying groups, and one-way ANOVA.

\section{Results}

Of the 150 subjects selected, $64 \%$ were female and 36\% male, $65 \%$ were East Indian descent, 30\% African descent and 5\% identified as mixed ethnicity. The majority of the population $(65 \%)$ was between the ages $54-69$ years, followed by $30 \%$ between $20-49$ years and $15 \%$ were more than 74 years (Figure 1A).

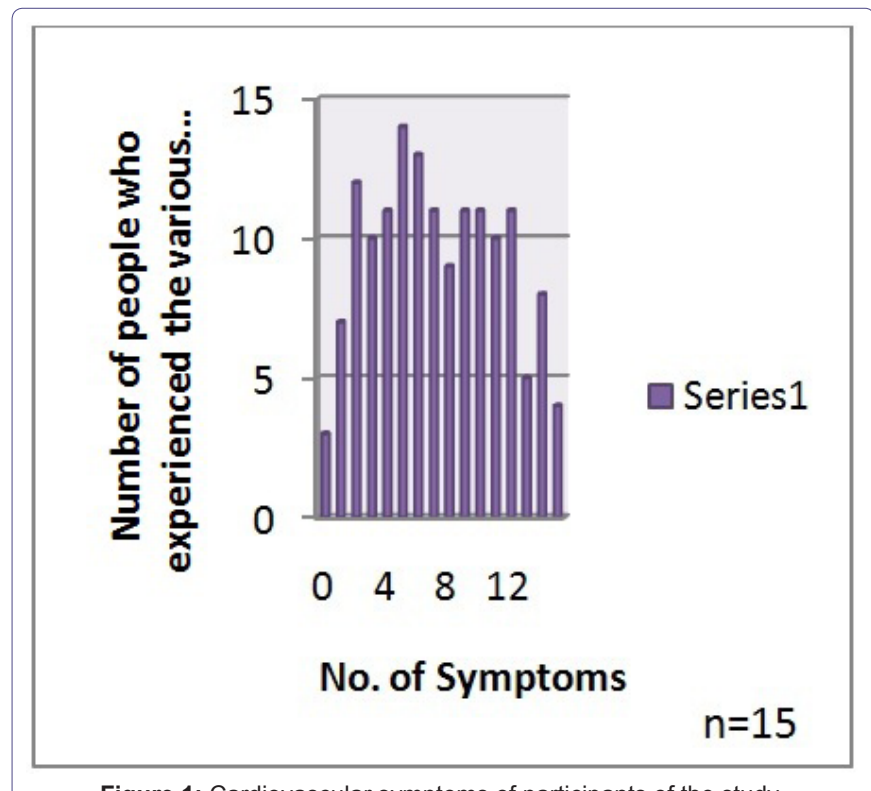

Figure 1: Cardiovascular symptoms of participants of the study.

The majority of the members $37 \%$ and $33 \%$ were found to be overweight and obese, respectively, whereas $28 \%$ subjects had a normal BMI. Of the participants, $98 \%$ experienced one or more CVD symptoms. Whereas $9 \%$ of the study population suffered from all the five symptoms (Figure 1).

Approximately $50 \%$ of individuals had scores within the range 51-60. As scores increase, the number of persons increased, up to a total score of 60 , after which the number of persons decreased (Figure 2). Half of the participants exhibited reduced sleep quality scores. Participants of $68 \%$ had $\mathrm{HbAlc}$ values within the $3^{\text {rd }}$ range (> 6.4\%), $21 \%$ had normal values, and $11 \%$ had lower than normal scores $(<5.7 \%)$ (Table 1$)$.

Our research also found that persons of African descent had a mean systolic pressure of $157.7 \pm 5.346$ while those of East Indian descent had an average of $140.9 \pm 2.683$ (Table 2).

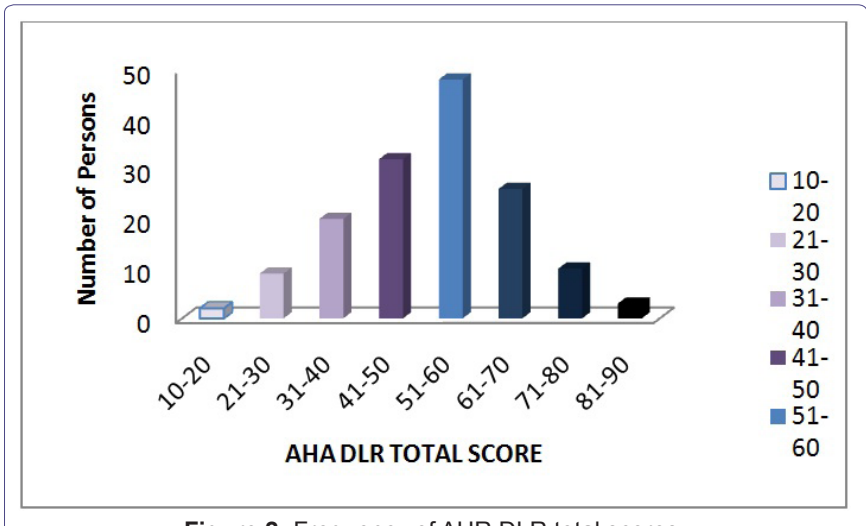

Figure 2: Frequency of AHR DLR total scores.

Framingham values, when compared with lifestyle factors, revealed a relationship between them. It was found that persons with allow risk of CVD scored on average $53.7 \pm 1.407$ in the AHA test and those with an intermediate risk of CVD scored an average of $47.5 \pm 2.277$. No significance was seen between high Framingham scores and AHA total. However, persons having a high risk of CVD had and overall lower average AHA scores (Table 3).

The study also revealed a significant relationship between systolic blood pressure and quality of sleep. For sleep score in the $1^{\text {st }}$ range $(<5)$ there was an average blood pressure of $152.83 \pm 3.855$ while those within the $2^{\text {nd }}$ range $(>5)$ had an average blood pressure of $141.19 \pm 3.100$ (Table 2).

\section{Discussion}

It was found that most of the people who took part in the study were of East Indian decent. That is, approximately $65 \%$ of the diabetics were East Indian. This is significant as a 1998 study done in Trinidad found that there was a greater prevalence of diabetes in East Indians. When comparing systolic blood pressure across the different ethnicities evaluated in the study, it was found that persons of African descent had significantly higher systolic blood pressure than those of East Indian descent. When ethnicity was compared with HbAlc, BMI, sleep score, and AHA total it was found to be insignificant.

Most of the males in the study had and intermediate risk for CVD within ten years. Where approximately $50 \%$ of the men had between $10-20 \%$ risk of contracting CVD. When Framingham values for menwere compared to lifestyle characteristics, a significant relationship was seen between the $1^{\text {st }}$ and $2^{\text {nd }}$ ranges. Even though there was no significant correlation between high Framingham scores and AHA totals, it is still seen that persons having a high risk of CVD had overall lower average AHA score. Therefore, we can deduce that the lower the lifestyle scores, the higher the ten-year risk for CVD may be. For females, the same trends were found. However, approximately $75 \%$ of the women in the study had a small percentage chance of developing CVD.

CVD is the leading cause of mortality in type2 diabetics [13]. This finding is also reflected in our research which revealed that $50 \%$ of males were at a moderate risk of coronary heart disease, and $25 \%$ of female participants were within intermediate and high-risk levels. They also highlight the fact that diabetes predisposes a person to hypertension which in turn increases the risk of developing cardiovascular diseases [13]. Our research concurs this finding. Participants of $52 \%$ were hypertensive which resultantly increased their Framingham's index score. 
Citation: Nayak BS, Sakhamuri SM, Moore I, Jordan D, Cuffie E, et al. (2016) Relationship of Lifestyle, Quality of Sleep with Type 2 Diabetes and Cardiovascular Risk - A Trinidadian Study. J Diabetes Metab Disord 3: 013.

\begin{tabular}{|l|c|c|c|c|c|c|c|c|c|}
\hline \multirow{3}{*}{ HbA1C } & Age & Ethnicity & Gender & Systolic BP & AHA Total & Sleep Score & $\begin{array}{c}\text { BMI } \\
\text { Percentage }\end{array}$ \\
\cline { 2 - 10 } & 1 & $61.18 \pm 2.82$ & $1.35 \pm 0.11$ & $1.59 \pm 0.12$ & $161.41 \pm 11.04$ & $50.29 \pm 2.65$ & $6.76 \pm 1.33$ & $26.1 \pm 1.07$ & $10.6 \pm 2.48$ \\
\cline { 2 - 10 } & 2 & $63.39 \pm 2.06^{*}$ & $1.48 \pm 0.11$ & $1.58 \pm 0.09$ & $142.74 \pm 3.99$ & $53.16 \pm 2.45$ & $7.03 \pm 0.82$ & $28.5161 \pm 1.04$ & $10.55 \pm 1.4$ \\
\cline { 2 - 9 } & 3 & $55.87 \pm 1.21^{*}$ & $1.37 \pm 0.05$ & $1.67 \pm 0.04$ & $145.90 \pm 2.88$ & $51.04 \pm 1.44$ & $5.90 \pm 0.37$ & $27.8431 \pm 0.62$ & $8.03 \pm 0.75$ \\
\hline
\end{tabular}

\begin{tabular}{|c|c|c|c|c|c|c|c|}
\hline \multirow{3}{*}{ Ethnicity } & & Systolic BP & $\begin{array}{c}\text { Framingham } \\
\text { Percentage }\end{array}$ & HbA1c & BMI & Sleep Score & AHA Total \\
\cline { 2 - 8 } & 1 & $140.9 \pm 2.68^{*}$ & $9.4 \pm 0.83$ & $7.8 \pm 0.02$ & $27.7 \pm 0.63$ & $6.5 \pm 0.45$ & $50.5 \pm 1.39$ \\
\cline { 2 - 8 } & 2 & $157.7 \pm 5.34^{*}$ & $8.2 \pm 1.14$ & $7.9 \pm 0.34$ & $27.8 \pm 0.08$ & $5.3 \pm 0.53$ & $52.2 \pm 2.05$ \\
\cline { 2 - 8 } & 3 & $162.8 \pm 11.33$ & $3.43 \pm 1.17$ & $7.8 \pm 0.18$ & $27.8 \pm 1.56$ & $7.4 \pm 1.31$ & $58.7 \pm 6.29$ \\
\hline
\end{tabular}

${ }^{*}=\mathrm{P}<0.05$

\begin{tabular}{|c|c|c|c|c|c|c|}
\hline \multirow{4}{*}{$\begin{array}{c}\text { Framingham estimated } \\
\text { 10-year risk percentage }\end{array}$} & & Ethnicity & BMI & AHA Total & HbA1c \\
\cline { 2 - 6 } & 1 & $1.46 \pm 0.06$ & $27.7 \pm 0.6$ & $53.77 \pm 1.4^{*}$ & $7.9 \pm 0.2$ \\
\cline { 2 - 7 } & 2 & $1.25 \pm 0.06$ & $28.6 \pm 0.8$ & $47.55 \pm 2.2 \#$ & $7.6 \pm 0.3$ \\
\hline
\end{tabular}

Table 3: Mean \pm SE of the different Framingham estimated 10-year risk percentage.

${ }^{*}=\mathrm{P}<0.05$

Reduced sleep was directly related to high weights [14]. This was evident in our research since there was significance between BMI and PSQI sleep score. On the other hand, Iyer reported that persons with diabetes have complaints with either initiating sleep or difficulty in maintaining sleep. This finding was also highlighted in our research where $50 \%$ of our participants has poor sleeping habits according to PSQI. The researchers also stated that age was a direct factor leading to diabetes [14]. This, however, was not found in our research.

Lichtenstein et al., stated that an improvement in diet and lifestyle is proportional to the prevention of CVD [15]. In our study, we found that diabetes was directly related to the development of CVD, as was evident from the Framingham scores and number of CVD symptoms participants' described. Improving lifestyle, through an increase in vegetable and fruit intake, an increase in physical exercise and a decrease in smoking and alcohol consumption would lead to a reduction in CVD risk and, therefore, aid in the control of diabetes [16].

The Framingham points score is universally used to predict the 10 -year risk for CVD in both men and women. The Framingham score is essential in helping clinicians predict the risk of patients developing coronary heart disease and thereby provide optimal treatment for these patients. It was also found to be paramount in predicting patients with other CVDs. This fact was proven in a study, which suggested that Framingham risk score could also be useful in predicting intermediate-term myocardial infarction risk among patients who recently had strokes [17]. Framingham has proven to be effective in accurately estimating the coronary heart disease risk in both men and women of different ethnic backgrounds though sometimes there might be an over estimation as shown [12]. The Framingham Score was used in our research over other cardiovascular predictors such as Joint British Societies and Assign [18]. The Joint British Societies do not incorporate diabetics' risk factors which are built in Framingham and were an integral component of our researcher since it was based on diabetics [18].
Since sleep quality is a contributing factor towards the prevalence of diabetes, a parameter such as the (PSQI) was developed to measure a person's sleeping patterns. PSQI is an index that measures subjective sleep [19]. PSQI scores were found to be useful in predicting depression and psychosocial stress [20]. It also enables the differentiation between 'good' and 'poor' sleepers [19]. PSQI, though is used in measuring sleep scores of older persons, was used in our research to encompass individuals ranging from 17 years to 81 years old [19].

AHA diet and lifestyle recommendations are intended to aid in the reduction of CVD risk. In our research, we used the modified AHA DLR Adherence Index T2DM. The index, therefore, aims towards the achievement of a desirable lipid profile, normal blood pressure, normal blood glucose, improved physical activity and decreased tobacco usage which is of most significance in diabetes management [15].

The indices used in the study can be of great benefit clinically. These indices can prove vital in making quick yet efficient diagnoses in clinical settings. By having access to data targeted to specific areas of the patients' lifestyle, clinicians obtain a clearer augmented image of the patients' history, thereby simplifying the diagnostic process. Based on the indices, the clinician can narrow the spectrum of probable diagnoses by analyzing and incorporating risk factors as it relates to patient health. The indices are also beneficial in measuring health improvement or decline, as well as identifying a possible treatment plan.

Our study showed that the lower the lifestyle scores, the higher the ten-year risk for CVD. Persons with lower sleep scores were found to have higher systolic blood pressures that those with higher sleep scores, revealing a link between sleep habits and CVD in diabetes.

The study had some limitations, especially when pieces of information were missing when we accessed the patient records. This 
study was conducted by year two medical student as a part of their Pubic health project and therefore due to a short period of six months researchers were not able to collect more data and follow up the diabetic patients.

Lack of sleep may be a contributing factor to insulin resistance and cause T2DM. It is also found that insufficient sleep is a risk factor for leptin deficiency which increases the urge for carbohydrates consumption regardless of the quantity in the body [6].

Lifestyle plays an integral role and incorporates factors such as diet, exercise, stress levels, as well as the use of alcohol and cigarettes. These have been linked to both predisposition and exacerbation of T2DM [1]. Persons, who consume high levels of fats and carbohydrates regularly, often develop metabolic syndrome. Elevated serum fatty acids and triglycerides have been shown to decrease the insulin sensitivity of cells and are also essential components in the development of obesity [7]. Also, high carbohydrate intake will result in consistently high blood a glucose level which is the chief contributing factor towards developing insulin resistance [4].

Regular physical activity improves the body's response to insulin as this allows more glucose to be broken down by metabolism due to the increased glucose demand of the body during exercise [1]. Ultimately, a lack of physical activity will reduce the utilization of glucose, contributing to high blood glucose levels [5].

As mentioned above, quality of sleep and lifestyle are associated to diabetes. Poor sleep may contribute to raising the risk of developing obesity, diabetes and cardiovascular disease. Poor sleep has been shown to be associated with elevations in inflammatory markers associated with obesity. These elevations have been connected with an increased risk for CVD [6].

\section{Acknowledgement}

We thank Dr. Shamjeet Singh, Dr. Shalini Pooransingh for their help. Authors would also like to thank the chairpersons of the all the regional health authorities for allowing us to access their patient data. We also thank all the participants who volunteered for our study.

\section{Conflict of Interest}

None

\section{Author Contributions}

All authors listed in this article are equally responsible for designing and conducting this study.

\section{References}

1. Kalyani RR, Brathwaite B (2010) Epidemiology of type 2 diabetes: Trinidad and Tobago specific information. Introduction to diabetes, Diabetes Guide, Point-of-care-information Technology, John Hopkins POC-IT Centre, USA.

2. Saudek C, Kalyani RR, Khan C, Brennan N (2010) Trinidad and Tobago specific information. Introduction to diabetes, Diabetes Guide, Point-of-care-information Technology, John Hopkins POC-IT Centre, USA.
3. The Ministry of Health (2010) World diabetes day. Government of the republic of Trinidad and Tobago, The Ministry of health, Trinidad and Tobago, West Indies.

4. Dewan NA (2016) Sleep, metabolic disturbance and diabetes mellitus: Are they linked? Chest, USA.

5. Insulite Laboratory (2012) Metabolic syndrome and pre and type 2 diabetes. Insulite Laboratory, USA.

6. Cappuccio FP, D'Elia L, Strazzullo P, Miller MA (2010) Quantity and quality of sleep and incidence of type 2 diabetes: a systematic review and meta-analysis. Diabetes Care 33: 414-420.

7. Bhupathiraju SN, Lichtenstein AH, Dawson-Hughes B, Tucker KL (2011) Adherence index based on the AHA 2006 diet and lifestyle recommendations is associated with select cardiovascular disease risk factors in older Puerto Ricans. J Nutr 141: 460-469.

8. Kuroki Y, Kanauchi K, Kanauchi M (2012) Adherence index to the American Heart Association Diet and Lifestyle Recommendation is associated with the metabolic syndrome in Japanese male workers. Eur J Intern Med 23: 199203.

9. Carney S, Koetters T, Cho M, West C, Paul SM, et al. (2011) Differences in sleep disturbance parameters between oncology outpatients and their family caregivers. J Clin Oncol 29: 1001-1006.

10. Taibi DM, Vitiello MV (2011) A pilot study of gentle yoga for sleep disturbance in women with osteoarthritis. Sleep Med 12: 512-517.

11. Neal AV, Hwalek MA, Scott RO, Sengstock MC, Stahl C (1991) Validation of the Hwalek-Sengstock elder abuse screening test. Journal of Applied Gerontology 10: 406-418.

12. D'Agostino RB Sr, Grundy S, Sullivan LM, Wilson P (2001) Validation of the Framingham coronary heart disease prediction scores: results of a multiple ethnic groups investigation. JAMA 286: 180-187.

13. Pyörälä K, Laakso M, Uusitupa M (1987) Diabetes and atherosclerosis: an epidemiologic view. Diabetes Metab Rev 3: 463-524.

14. Iyer SR (2012) Sleep and type 2 diabetes mellitus- clinical implications. J Assoc Physicians India 60: 42-47.

15. American Heart Association Nutrition Committee, Lichtenstein AH, Appel LJ, Brands M, Carnethon M, et al. (2006) Diet and lifestyle recommendations revision 2006: a scientific statement from the American Heart Association Nutrition Committee. Circulation 114: 82-96.

16. Howard AA, Arnsten JH, Gourevitch MN (2004) Effect of alcohol consumption on diabetes mellitus: a systematic review. Ann Intern Med 140: 211-219.

17. Towfighi A, Markovic D, Ovbiagele B (2012) Utility of Framingham coronary heart disease risk score for predicting cardiac risk after stroke. Stroke 43: 2942-2947.

18. Payne R (2010) The University of Edinburgh Cardiovascular Risk Calculator.

19. Ciccone MM, Scicchitano P, Cameli M, Cecere A, Cortese F, et al. (2014) Endothelial Function in Pre-diabetes, Diabetes and Diabetic Cardiomyopathy: A Review. J Diabetes Metab 5: 364-366.

20. Mondal P, Gjevre J, Taylor-Gjevre RM, Lim HJ (2013) Relationship between the Pittsburgh sleep quality index and the Epworth sleepiness scale in a sleep laboratory referral population. Nat Sci Sleep 5: 15-21. 